\title{
US-guided interventional joint procedures in patients with rheumatic diseases-When and how we do it?
}

\author{
B. Gonçalves ${ }^{\mathrm{a}, *}$, C. Ambrosio ${ }^{\mathrm{b}}, \mathrm{S}$. Serra ${ }^{\mathrm{b}}$, F. Alves ${ }^{\mathrm{a}}$, A. Gil-Agostinho ${ }^{\mathrm{a}}$, F. Caseiro-Alves $^{\mathrm{a}}$ \\ a Department of Radiology, Hospitais da Universidade de Coimbra - HUC, Coimbra, Portugal \\ ${ }^{\mathrm{b}}$ Department of Rheumatology, Hospitais da Universidade de Coimbra - HUC, Coimbra, Portugal
}

\section{A R T I C L E I N F O}

\section{Article history:}

Received 24 January 2010

Accepted 7 April 2010

\section{Keywords:}

Rheumatic diseases

Interventional radiology

Ultrasound

Joint steroid injections

Radiosynoviorthesis

Arthrocentesis

Biopsy

\begin{abstract}
A B S T R A C T
Objective: To describe the main indications and the technical steps to perform ultrasound guided procedures in patients with rheumatic diseases. To access procedures accuracy, safety and effectiveness. Materials and methods: 27 patients with pain related to articular complications of rheumatic diseases and according to previous radiographic or US exam were submitted to several US-guided procedures. $42 \%$ of patients $(n=11)$ had rheumatoid arthritis, $11 \%(n=3)$ spondyloarthropathies, $18 \%(n=5)$ psoriatic arthritis, 15\% ( $n=4)$ undifferentiated arthritis, 3\% $(n=1)$ Sjögren syndrome and 11\% $(n=3)$ had gout.

Described procedures are synovial biopsies, intra-articular injections of corticosteroids, radiation synovectomy and synovial cysts drainage procedures. When a therapeutical procedure was made, patients were evaluated by 2 rheumatologists.

Corticosteroids used were Prednisolone and Triamcinolone. Yttrium-90 was used for synovectomy. Results: In all cases success was achieved with correct needle placement inside the joint. After injection/aspiration symptoms successfully solved with all patients improving their health status. No complications were recorded during follow-up period.

Conclusions: US-guidance is very reliable to afford a safety procedure always checking the injection, biopsy or aspiration. Guided-biopsy has high success rates obtaining several samples. Thus is also possible to use more powerful/long acting therapeutic drugs aggressive to extra-articular structures avoiding complications.
\end{abstract}

(c) 2010 Elsevier Ireland Ltd. All rights reserved.

\section{Introduction}

Ultrasound (US) guided interventional techniques are the most reliable way to access safely, quickly and accurately all the joints in the apendicular skeleton. In several cases rheumatic diseases need the help of ultrasonography to achieve a confident diagnosis characterizing the affected joints and evaluating disease activity. In a few cases the rheumatic disorder is only diagnosed by synovial biopsy or by joint fluid chemical and culture tests.

During chronic therapy rheumatic diseases course with acute exarcebations that must be promptly treated. During systemic therapy some joints also fail to respond and thus they benefit from intra-articular corticosteroid injections. US findings like synovial thickening, the presence of Power-Döppler signal (Fig. 1) or US-contrast enhancement indicate active synovitis (Fig. 2)

\footnotetext{
* Corresponding author at: Travessa dos Navegadores, Lote 1, 1.frente, 3030-065 Coimbra, Portugal.

E-mail address: belarmino.goncalves@gmail.com (B. Gonçalves).
}

and are well correlated with patient symptoms. US evaluation of the symptomatic joints improves therapeutic effectiveness of US-guided therapy by choosing the right place for injection. In those cases when a tendinitis or a tenosynovitis is present, a corticosteroid instillation in the tendon sheath can also reduce synovial inflammation and improving symptoms (Fig. 3) [1]. Yttrium-90 radiation synovectomy or radiosynoviorthesis (RSO) is another therapeutic technique based on an intra-articular image-guided infusion of a radionuclide. It intends to block intra-articular inflammation, reducing effusion, improving pain and joint swelling and thus restoring articular mobility (Fig. 4) [2-6].

Described techniques involve an approach with ultrasound guidance to place the needle intra-articularly. The following procedures also require a thorough knowledge of ultrasound anatomy to decide the optimum intra-articular site to perform the technique. Sonographic needle guidance significantly improves the performance and outcomes in a clinically significant manner [7].

This work describes intra-articular techniques guided by ultrasound at shoulder, elbow, wrist, metacarpophalangeal, knee, ankle and metatarsophalangeal joints. 


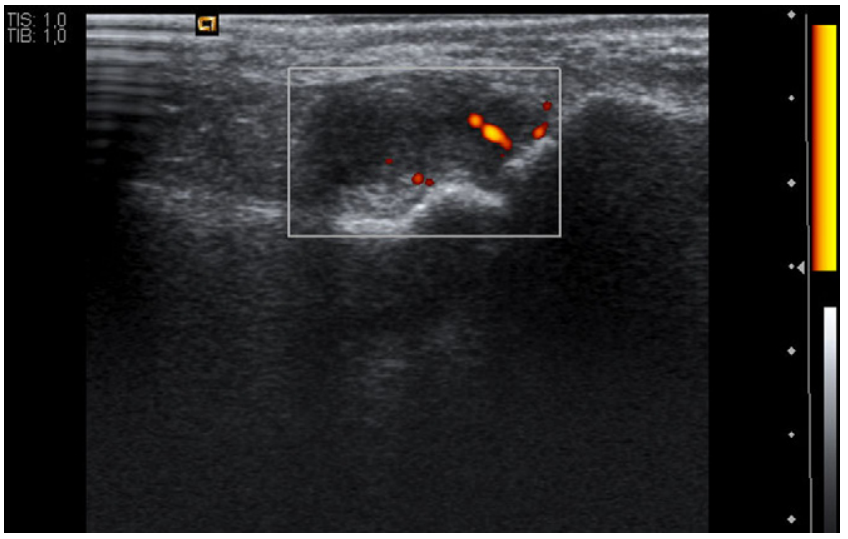

Fig. 1. Power-Döppler-US. Metatarsophalangeal joint showing synovial thickening and Döppler signal indicating active synovitis (Psoriatic Arthritis).

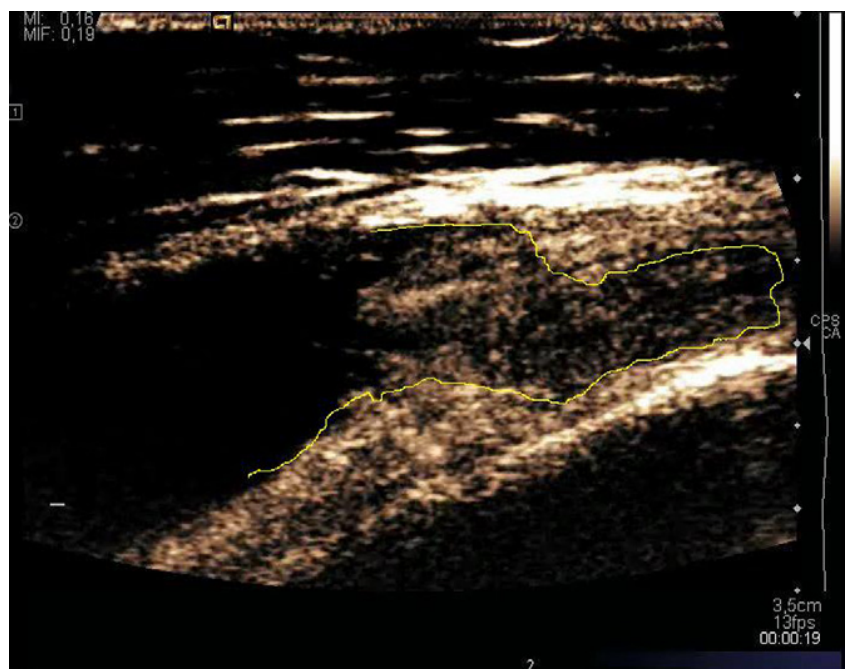

Fig. 2. Contrast Enhanced US (CEUS) of the supra-patelar recess showing early synovial enhancement and thus diagnosing active synovitis (Sjögren syndrome). Enhancement time is displayed in the right inferior corner of the image (19s). Enhancement region in the synovium is demarcated by the white line.

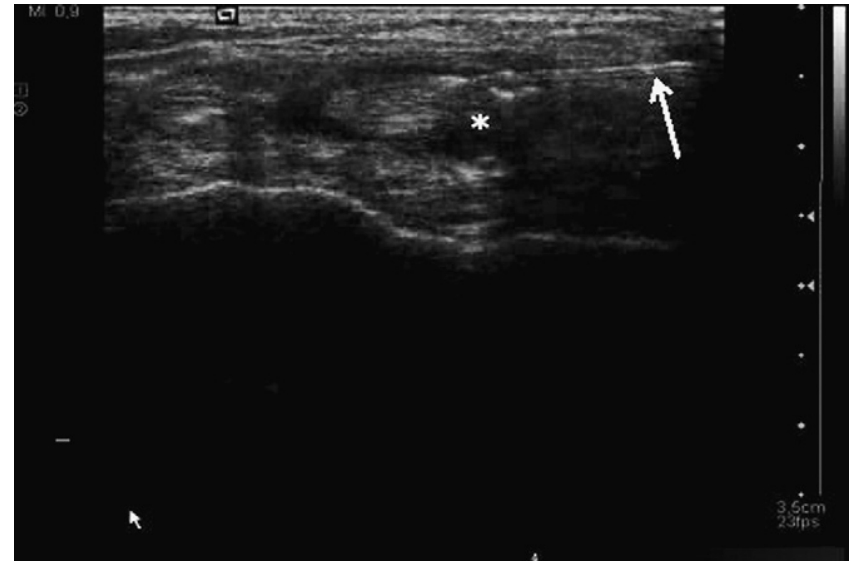

Fig. 3. Corticosteroid injection - patient with early Rheumatoid arthritis. US shows tenosynovitis in the tibialis posterior tendon. Methylprednisolone is being injected in the tendon sheath. $21 \mathrm{G}$ needle (arrow). The $\left(^{*}\right)$ indicates the air bubbles of the injection mixture.

\section{Material and methods}

\subsection{Patient selection}

27 patients with pain related to articular complications of rheumatic diseases and according to previous radiographic or US exam were submitted to the following US-guided procedures.

$42 \%$ of patients $(n=11)$ had rheumatoid arthritis, $11 \%(n=3)$ spondyloarthropathies, $18 \%(n=5)$ psoriatic arthritis, $15 \%(n=4)$ undifferentiated arthritis, 3\% $(n=1)$ Sjögren syndrome and 11\% $(n=3)$ had gout

\subsection{Procedures, articular puncture and joint specific features}

1. Synovial biopsy was made in 3 patients.

2. Arthrocentesis/cysts drainage was made in 8 patients.

3. Corticosteroid injections in 12 patients.

4. Radiation synovectomy (Yttrium-90) - US guided in 4 patients.
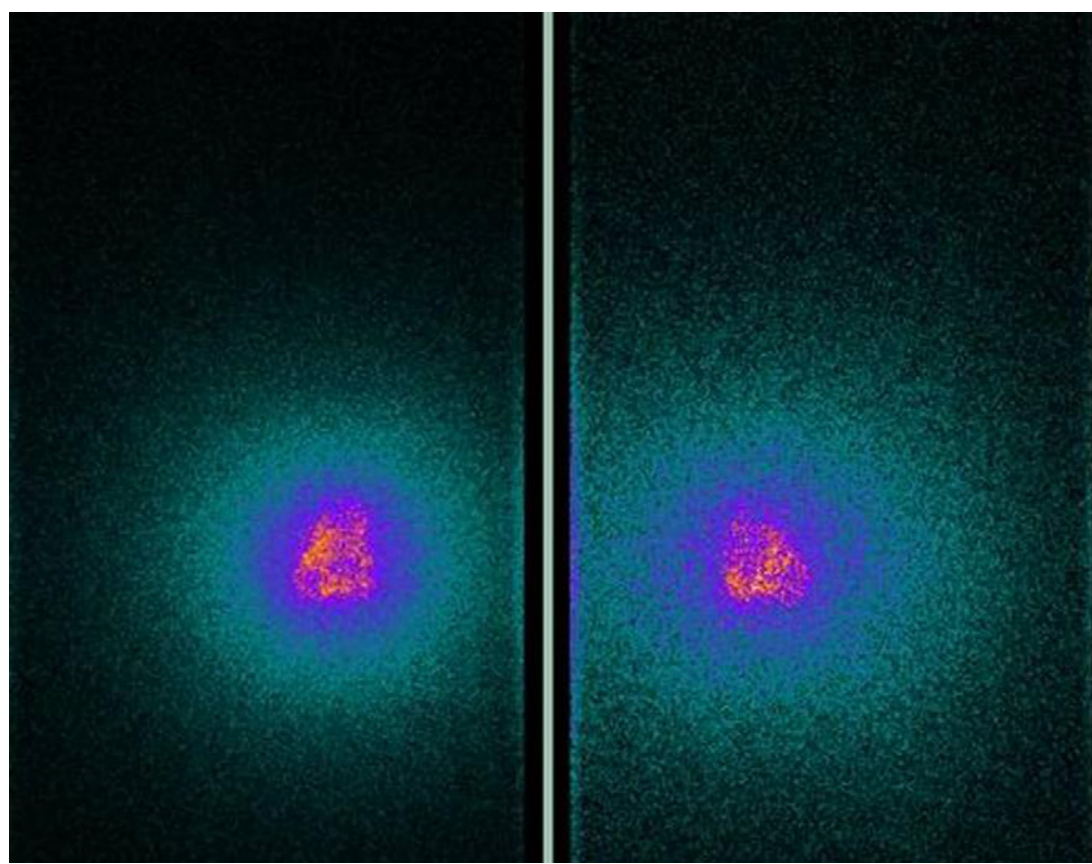

Fig. 4. Knee scintigram after injection of Yttrium-90 (anterior and posterior views). 


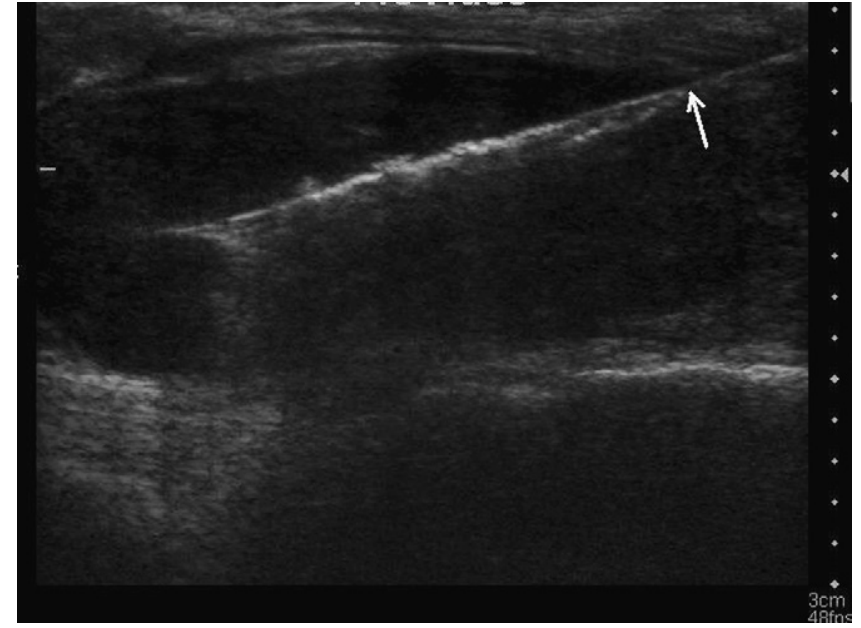

Fig. 5. Synovial biopsy - patient with an undifferentiated oligoarthritis. US shows the $18 \mathrm{G}$ needle (arrow) entering the thickened synovium (hypoechoic) in the suprapatellar recess.

All the procedures were done under strict aseptic conditions. It is very important to use all ultrasound anatomical landmarks. Puncture technique was performed according the radiologist experience and sometimes using a similar technique as in CT or MR arthrography [8]. Local skin anesthesia was performed almost in all procedures, particularly in the synovial biopsy. A lidocaine solution (1\%) was injected under the skin without air bubbles in the syringe to avoid ultrasound artifacts. The articular space is very wide in the knee, shoulder and elbow and relatively small in the wrist and phalangeal joints. We used a 21 gauge $\times 40 \mathrm{~mm}$ needle in the knee, elbow and in the ankle and a 23 gauge $\times 25 \mathrm{~mm}$ needle in the wrist, metacarpophalangeal and in the metatarsophalangeal joints. In the shoulder was used a spinal needle ( 21 gauge $\times 70 \mathrm{~mm}$ ). Yttrium90 radiosynoviorthesis was only made in the knee. Corticosteroids used were:

1. Triamcinolone hexacetonide is a relatively insoluble corticosteroid with a prolonged effect on tissue at the local injection site and the duration usually ranging from a few weeks to several months.

2. Methylprednisolone acetate was used for short-term administration and to tide the patient over an acute episode or exacerbation.

\subsection{Indications and techniques}

\subsubsection{Synovial biopsy}

The main indications to synovial biopsy were:

- To distinguish an inflammatory from an infectious arthropathy, particularly when there is an oligoarthritis.

- To make the differential diagnosis from other synovial thickening conditions like pigmented villonodular synovitis (PVNS) or a metabolic arthropathy (crystal deposition disease) (Fig. 5).

Material used:

- Sterile gloves, surgical gauze and drapes.

- Cleaning fluid - alcohol or iodine solution.

- 18-gauge thru-cut needle.

- $10 \mathrm{~mL}$ bottle of $0,9 \%$ sodium-chloride solution.

- $10 \mathrm{~mL}$ bottle of formaldehyde solution.

- Two $50 \mathrm{~mL}$ recipients for samples (one for sodium-chloride and other for formaldehyde solution).

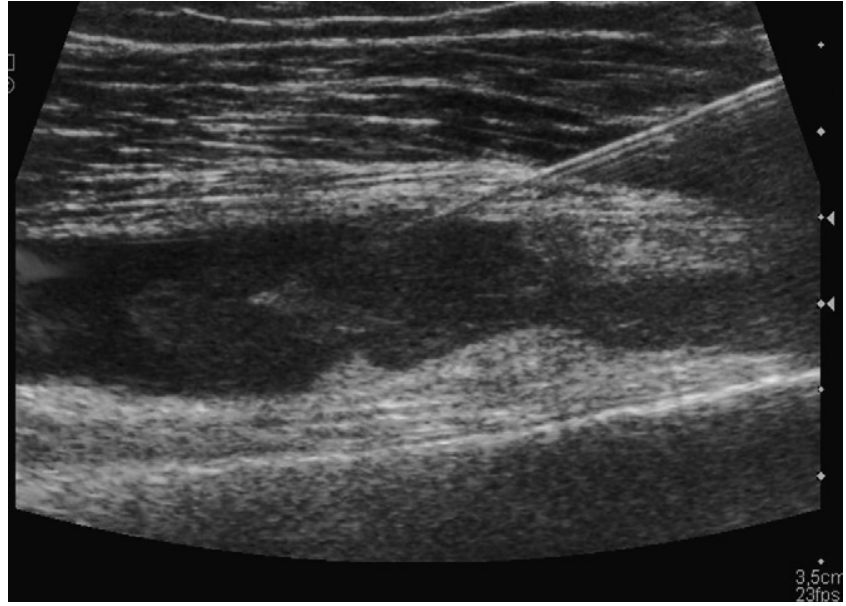

Fig. 6. Arthrocentesis - patient with an undifferentiated oligoarthritis. US shows the needle entering the supra-patellar recess to aspirate fluid.

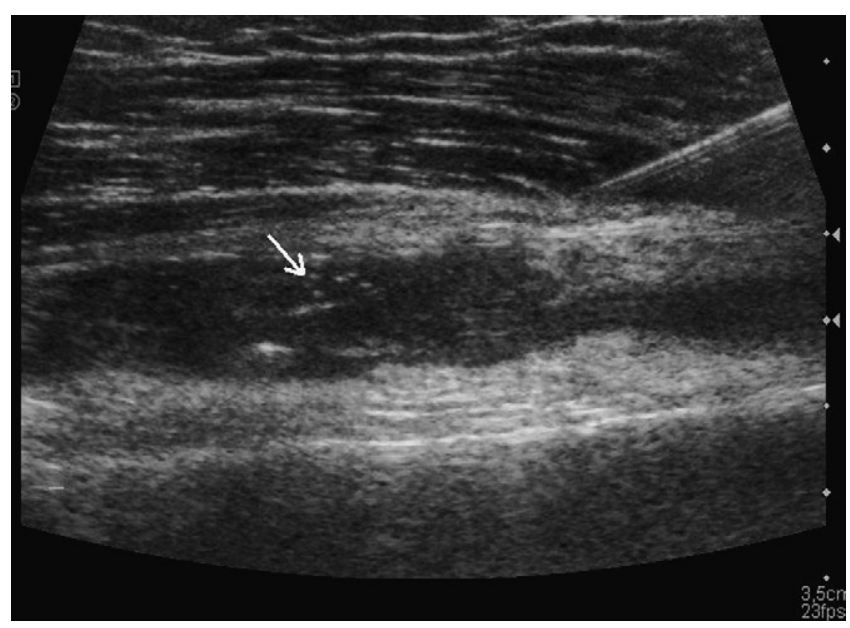

Fig. 7. Corticosteroid injection (triamcinolone) - patient with an undifferentiated oligoarthritis. US shows the needle entering the supra-patellar recess and bubbling effect of the drug. Air bubbles present in the injection mixture (arrow).

\subsubsection{Arthrocentesis/cysts drainage}

The main indications of guided arthrocentesis were (Fig. 6):

- To characterize the aetiology of a monoarthritis, particularly when there is a small amount of fluid.

- To complement synovial biopsy in the study of an oligoarthritis (for chemical and culture tests of the synovial fluid).

- To drain synovial cysts.

- To relief related symptoms.

Material used:

- Sterile gloves, surgical gauze and drapes.

- Cleaning fluid - alcohol or iodine solution.

- 21- or a 23-gauge needle according to the joint.

- $5 \mathrm{~mL}$ syringe(s).

- $50 \mathrm{~mL}$ sterile recipient for fluid.

\subsubsection{Corticosteroid injections}

The main indications of intra-articular corticosteroid injections were (Fig. 7):

- Symptomatic relief during joint acute exarcebations. 


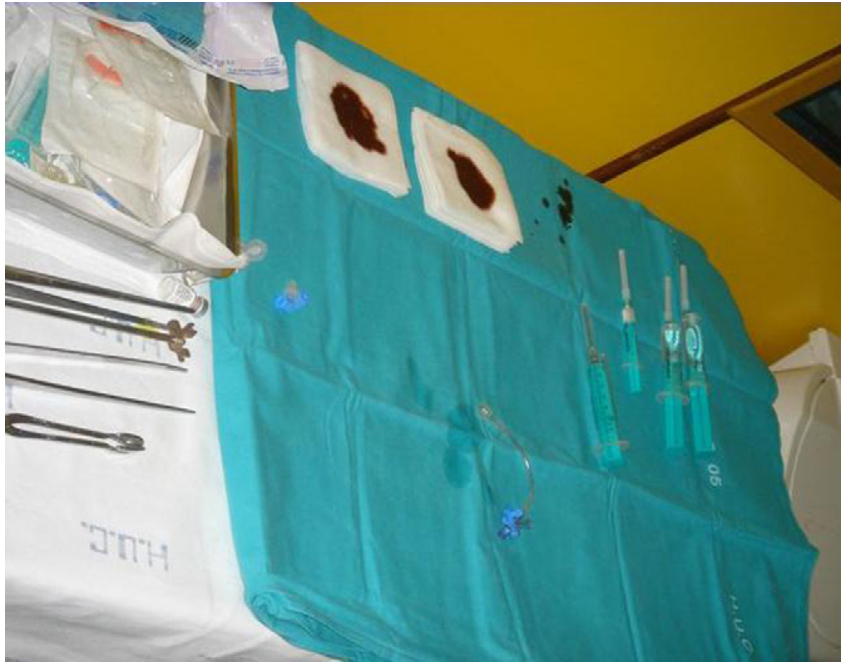

Fig. 8. Material used for radiosynoviorthesis (RSO).

- To treat synovial proliferation when it fails to respond to systemic therapy and thus reducing early joint destruction.

- To reduce relapsing effusions and to prevent synovial cysts recurrence.

Material used:

- Sterile gloves, surgical gauze and drapes.

- Cleaning fluid - alcohol or iodine solution.

- 21- or a 23-gauge needle according to the joint.

- One $2 \mathrm{~mL}$ syringe.

- $40 \mathrm{mg}$ of metilprednisolone (80 mg in bigger joints) or $20 \mathrm{mg}$ of triamcinolone hexacetonide ( $40 \mathrm{mg}$ in bigger joints).

\subsubsection{Radiosynoviorthesis - US guided}

The main indications for RSO-Y90 were:

- Persistent mono or oligoarthritis unresponsive to medical treatment over the last 6 months.

- Intolerance to medical treatment.

- Absence of response to an effective dose of intra-articular steroids.

- Absence of any absolute contraindication (pregnancy, lactation after pregnancy, skin infection, articular infection, bone fracture, popliteal cyst with rupture signs, severe hemarthrosis).

Material used:

- Sterile gloves, surgical gauze and drapes.

- Cleaning fluid - alcohol or iodine solution.

- $10 \mathrm{~mL}$ bottle of $0,9 \%$ sodium-chloride solution.

- $1 \mathrm{~mL}$ syringe (for Yttrium) and two $5 \mathrm{~mL}$ syringes (for sodiumchloride solution and for steroid).

- 21-gauge needle.

- 3-way stopcock with a $10 \mathrm{~cm}$ extension tube (Fig. 8).

- Yttrium-90 solution of $185 \mathrm{MBq}$ with isolating material around syringe (Fig. 9).

- $40 \mathrm{mg}$ of metilprednisolone (administered to reduce flare after radionuclide infusion).

\subsection{Clinical evaluation}

When a therapeutic procedure like corticosteroids injections or radiation synovectomy was made patients were evaluated by two

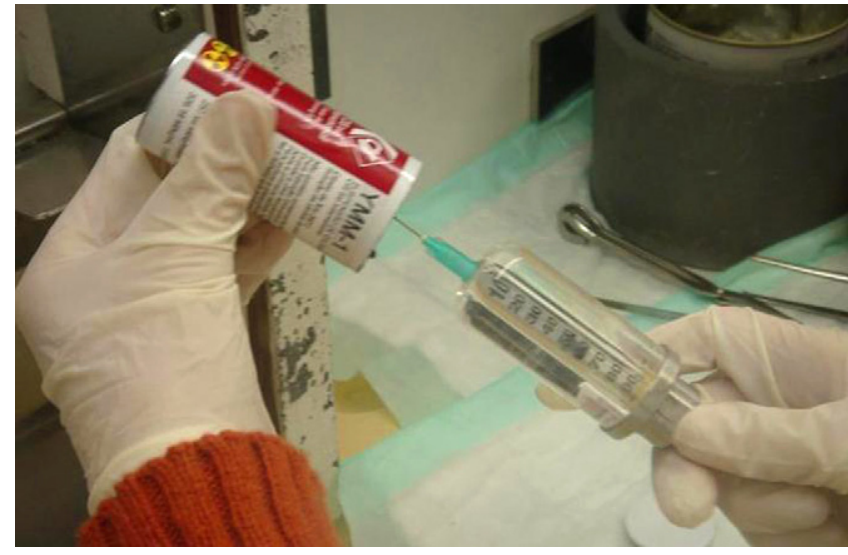

Fig. 9. Aspiration of Yttrium-90 from container. Isolating material around syringe.

How severe is your pain?

$$
\text { No pain }
$$

Worst pain imaginable

Fig. 10. Visual Analog Scale (VAS) for pain.

rheumatologists. Patients were evaluated at 3 weeks and 6 months. The clinical effect was assessed by evaluating stiffness, swelling, effusion and spontaneous or mechanical pain. A questionnaire scale was also applied - Visual Analog Scale (VAS) for pain (Fig. 10).

\section{Results}

\subsection{Procedure details}

With patient positioned on the ultrasound examination table and using an aseptic technique the surface puncture was cleaned (with alcohol or iodine solution). To the intra-articular placement of the needle all ultrasound anatomical landmarks were used, avoiding vessels, tendons or ligaments. Once the joint was entered and little resistance to injection was felt intra-articular placement was achieved. Because of the radiopharmaceutical drug (Yttrium-90) synovectomy required specific features for radiation protection.

\subsubsection{Shoulder}

Patient was placed seated with the shoulders adducted in neutral position. A posterior approach was used (Fig. 11). The US-probe was placed parallel to infraspinatus tendon with the tip of the needle advanced to the humeral head just laterally to the posterior labrum (Fig. 12). Other approaches can be used according to the radiologist experience $[9,10]$.

\subsubsection{Elbow}

Patient was placed seated with the elbow slightly extended. The probe was placed longitudinally showing anterior joint recess (Fig. 13). The tip of the needle advanced to the humeral trochlea (Fig. 14).

\subsubsection{Wrist}

Patient was placed seated with the elbow extended and wrist pronated on a table. Radiocarpal compartment was always used. The probe was placed longitudinally along the articular space along de radius and scapholunate space, about $1 \mathrm{~cm}$ distal to Lister's tubercle, with the needle making a proximal tilt of approximately $10-30^{\circ}$ and between the extensor pollicis longus and extensor digitorum communis (Figs. 15 and 16) [11]. 


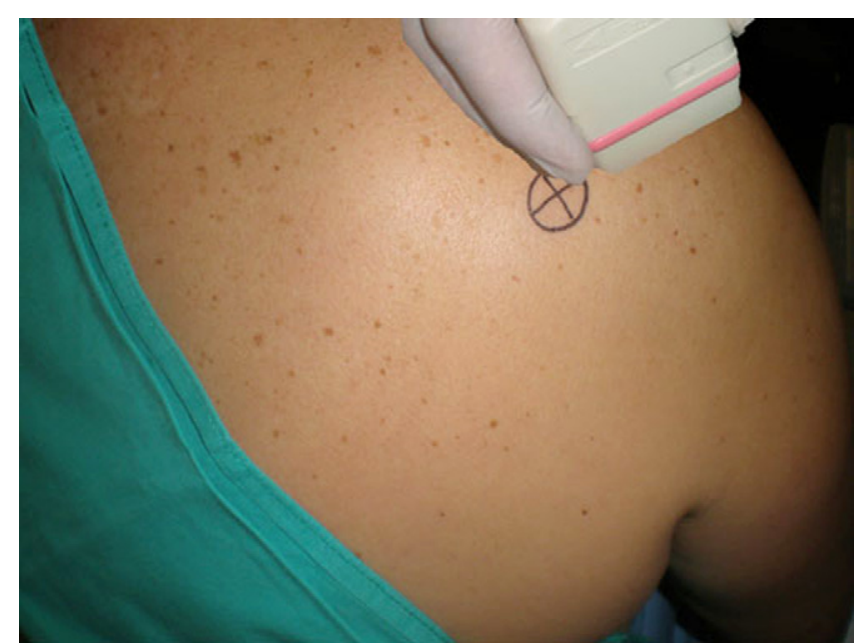

Fig. 11. US-probe position to puncture shoulder joint. A skin mark indicates puncture site to enter the gleno-humeral joint.

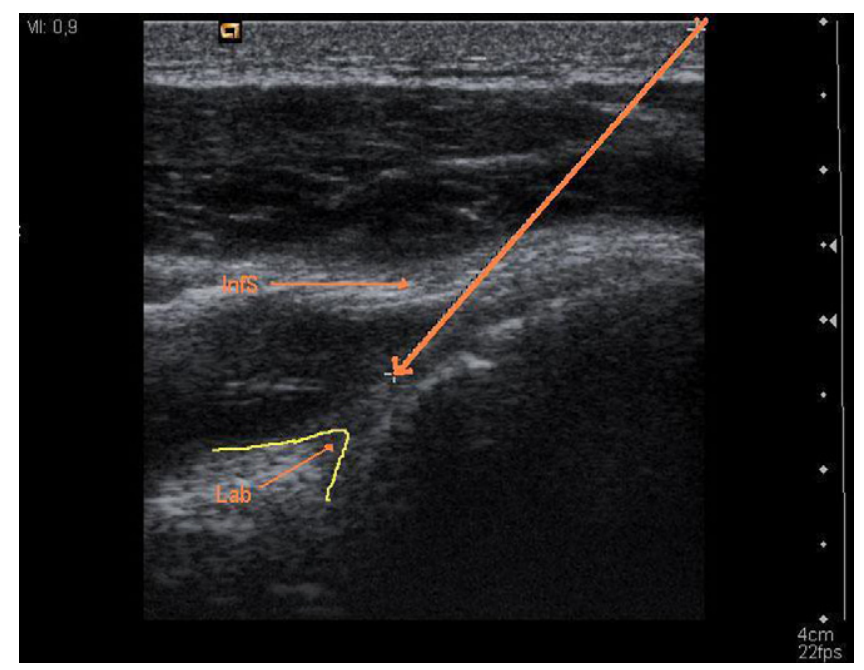

Fig. 12. US image shows the direction of the needle to enter the joint space between the humeral head an the labrum. Infraspinatus tendon (InfS) and Glenoid labrum $(L a b)$.

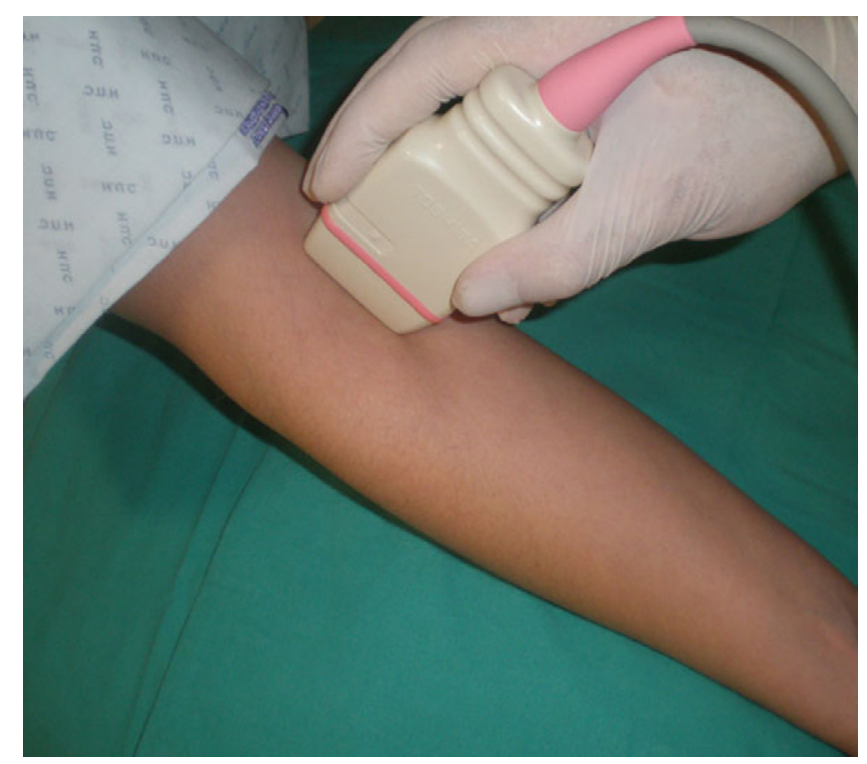

Fig. 13. US-probe position to enter the anterior recess of the elbow joint space.

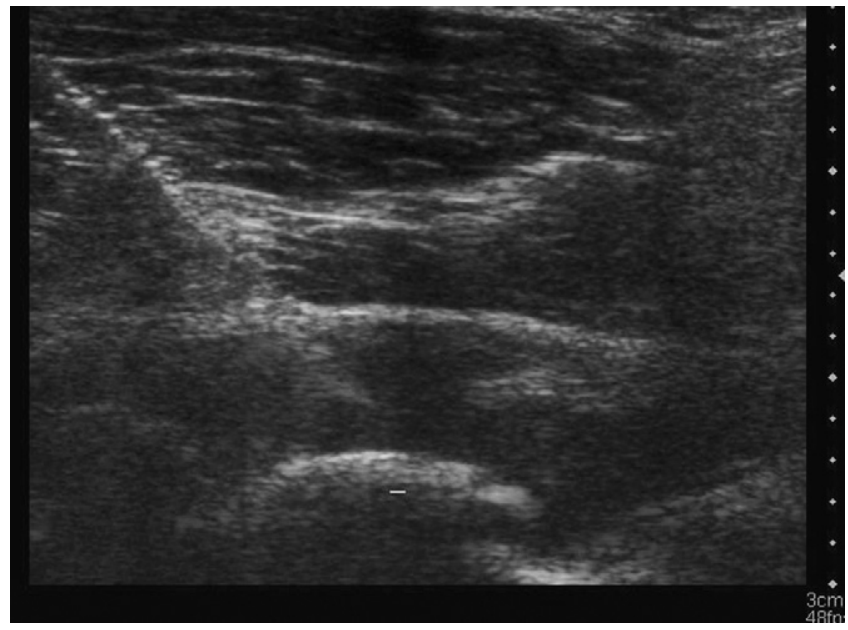

Fig. 14. Corticosteroid injection - patient with a Psoriatic arthritis. US shows marked synovial thickening and the tip of the needle in the anterior joint recess.

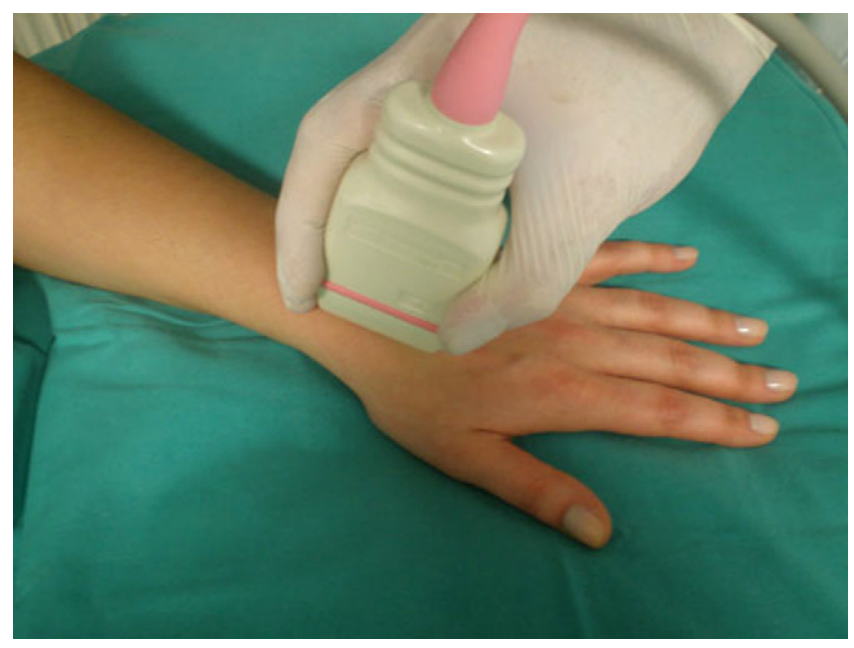

Fig. 15. US-probe position to enter the wrist joint space (radiocarpal compartment).

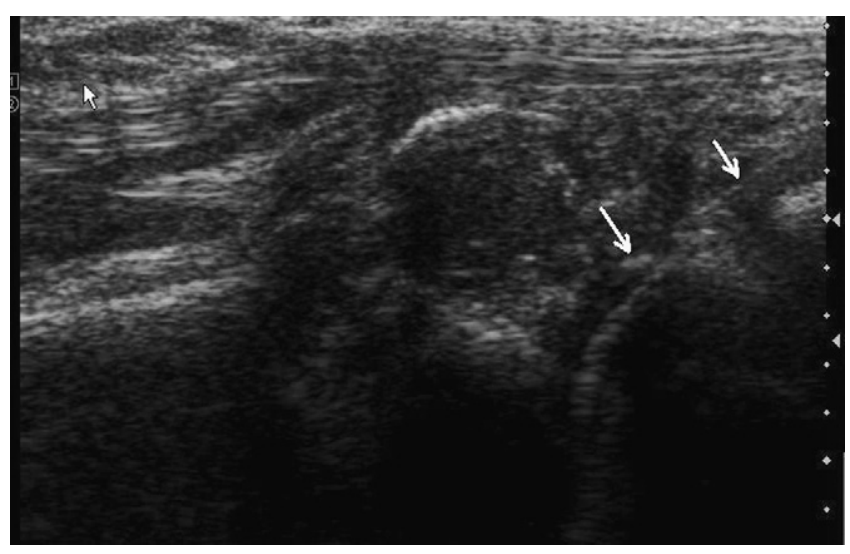

Fig. 16. Patient with a late Rheumatoid arthritis. US shows the needle advancing trough the radiocarpal compartment of the wrist (arrows).

\subsubsection{Metacarpophalangeal joints}

Patient was placed seated with the elbow extended, wrist pronated and fingers extended on a table. The probe was place in a longitudinal dorsoradial or dorsoulnar position along the articular space (Fig. 17). The needle was advanced avoiding extensor tendons. A subtle traction in opposite direc- 


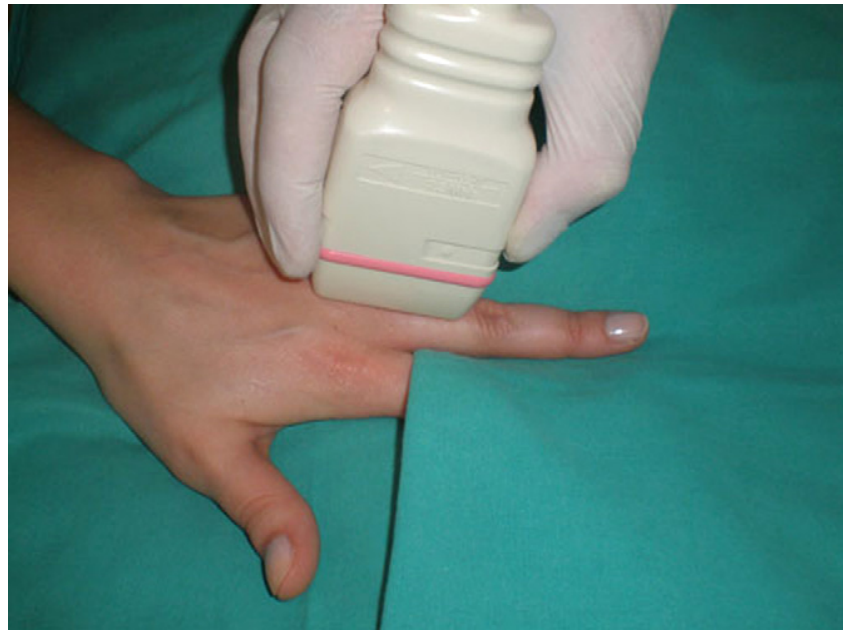

Fig. 17. US-probe position to puncture a metacarpophalangeal joint (3rd finger).

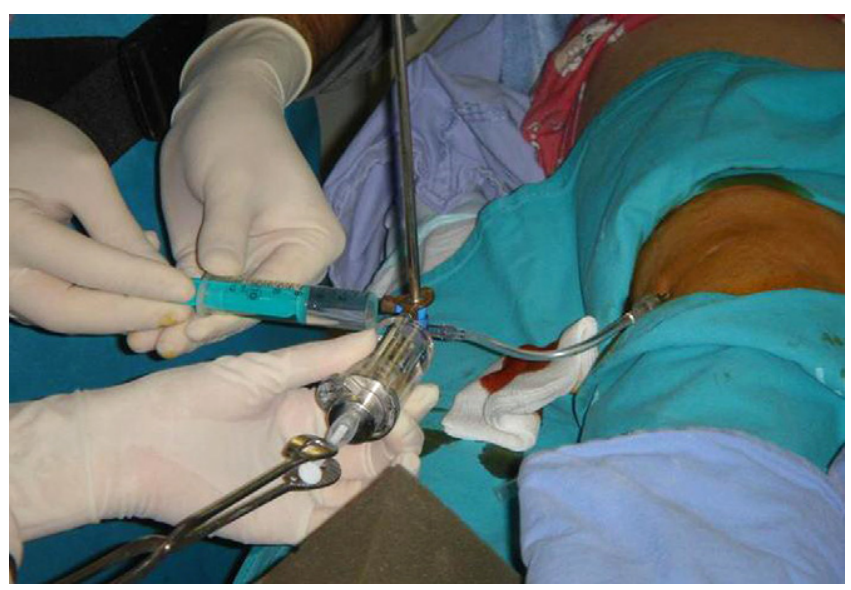

Fig. 19. Radiosynoviorthesis (RSO) procedure by lateral approach. Material used for radiation protection.

tion of the needle was helpful to slightly open the joint space.

\subsubsection{Knee}

Patient was placed seated on an examination table with the knee flexed $\left(30^{\circ}\right)$ and ankle extended. According to the procedure lateral (Fig. 19), anterior (above patella) (Figs. 7, 18, 20 and 21) or posterior approaches were used. When anterior approach was needed the needle was inserted into the soft tissue directed to the suprapatellar recess or when a posterior approach was needed the needle was directed to the popliteal cyst (Fig. 22) [12].

\subsubsection{Ankle}

Patient was placed seated on an examination table with the knee flexed $\left(45^{\circ}\right)$ and ankle extended. Placing the transducer probe longitudinally along the tibia and the talus, anteromedial approach was used with the needle between the anterior tibialis tendon and the medial malleolus (Fig. 23).

\subsubsection{Metatarsophalangeal joints}

Patient was placed seated on an examination table with the knee flexed $\left(45^{\circ}\right)$, ankle and fingers extended. The probe was place in a longitudinal dorso-lateral or dorso-medial position along the articular space (Fig. 24). The needle was advanced avoiding extensor tendons. A subtle traction in opposite direction of the needle was helpful to slightly open the joint space (Fig. 25).

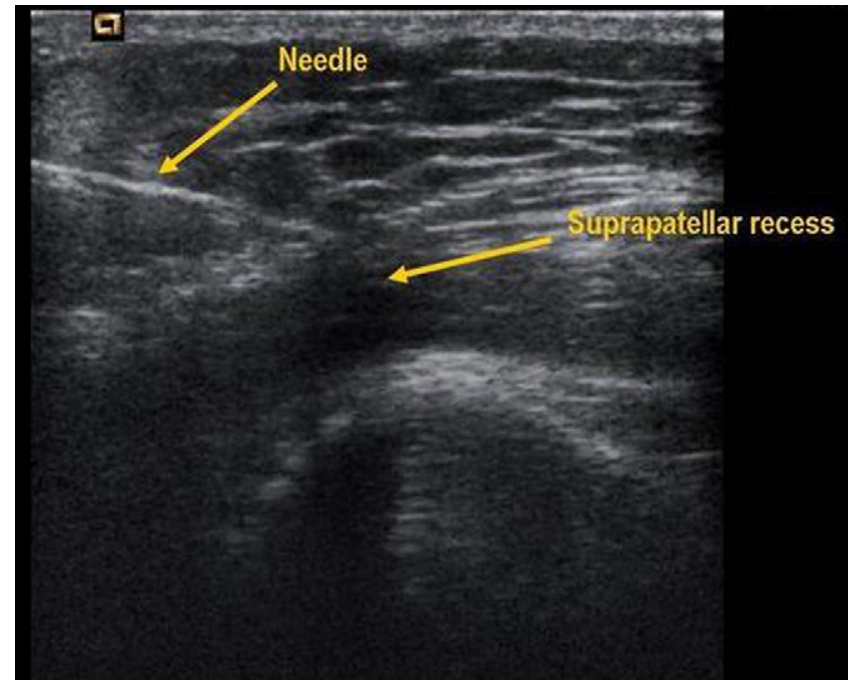

Fig. 18. Radiosynoviorthesis (Yttrium-90). US image shows the needle in the suprapatellar joint recess.

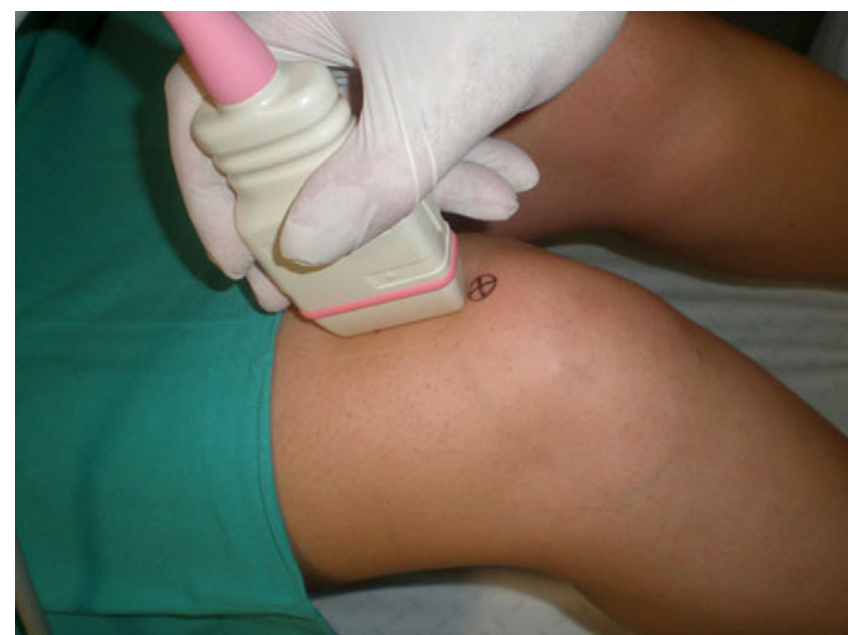

Fig. 20. US-probe position to enter the knee joint space (supra-patellar recess). A skin mark indicates puncture site.

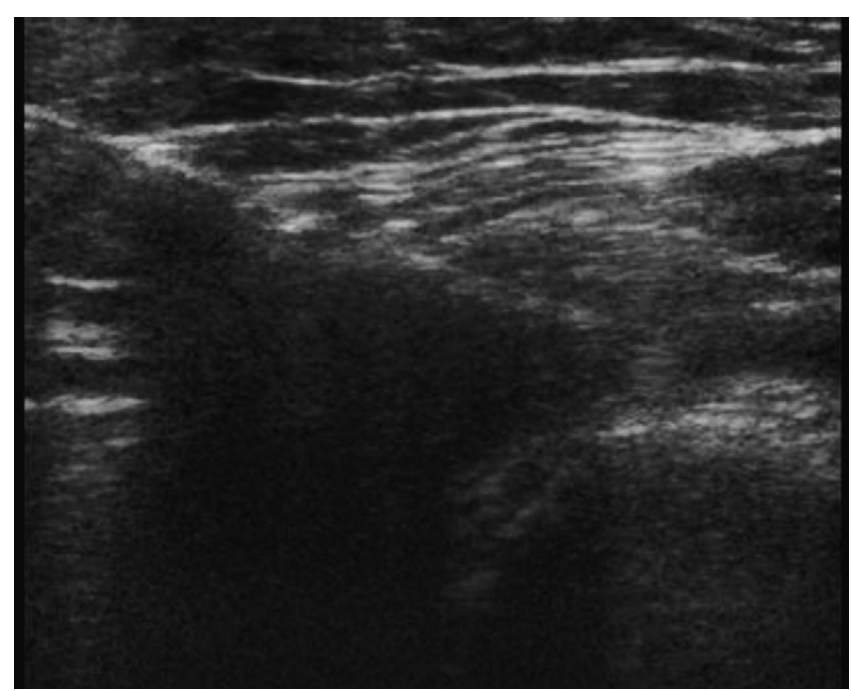

Fig. 21. Synovial biopsy - patient with an undifferentiated oligoarthritis. US shows the needle entering the thickened synovium in the supra-patellar recess. 


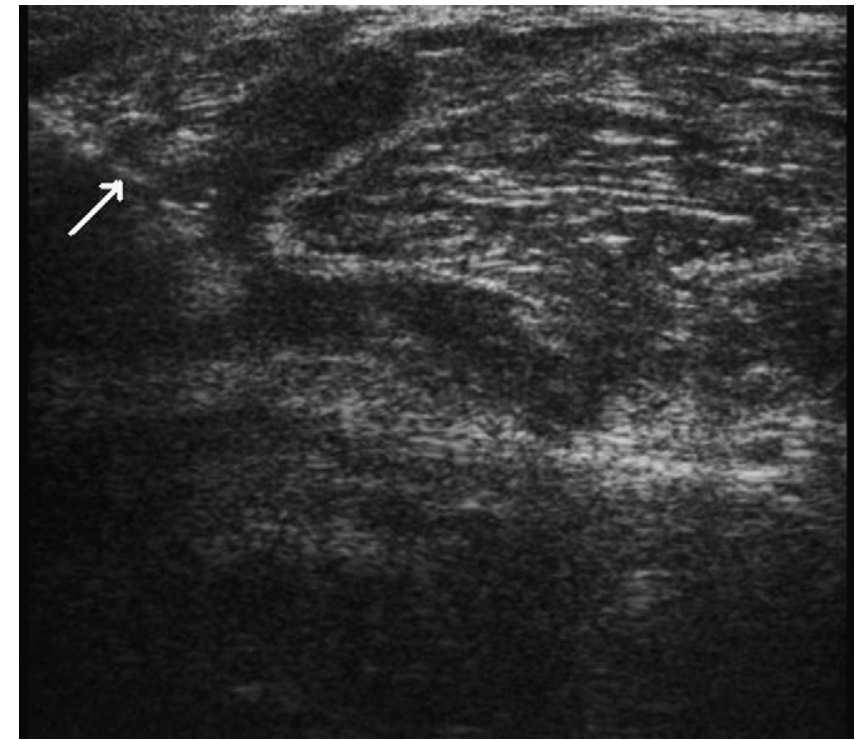

Fig. 22. Corticosteroid injection - patient with rheumatoid arthritis. US shows a popliteal cyst being injected with methylprednisolone after drainage. Arrow indicates the needle.

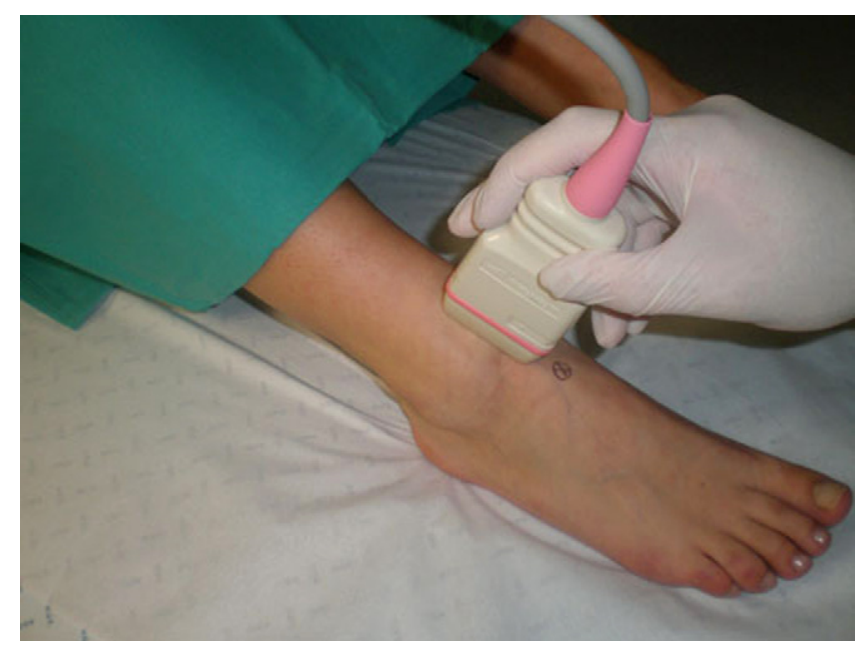

Fig. 23. US-probe position to enter the ankle joint space. A skin mark indicates puncture site.

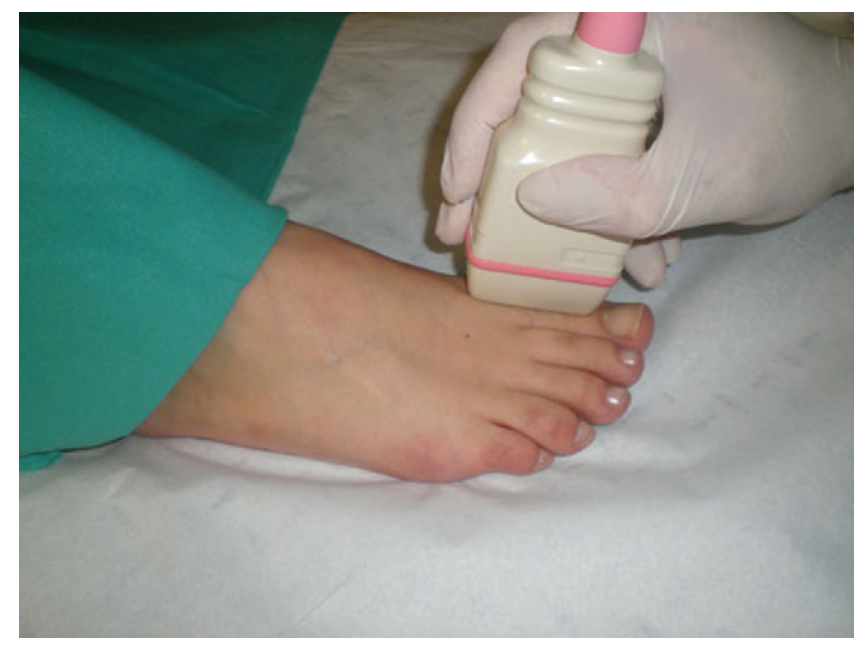

Fig. 24. US-probe position to puncture a metatarsophalangeal joint (1st finger shown).

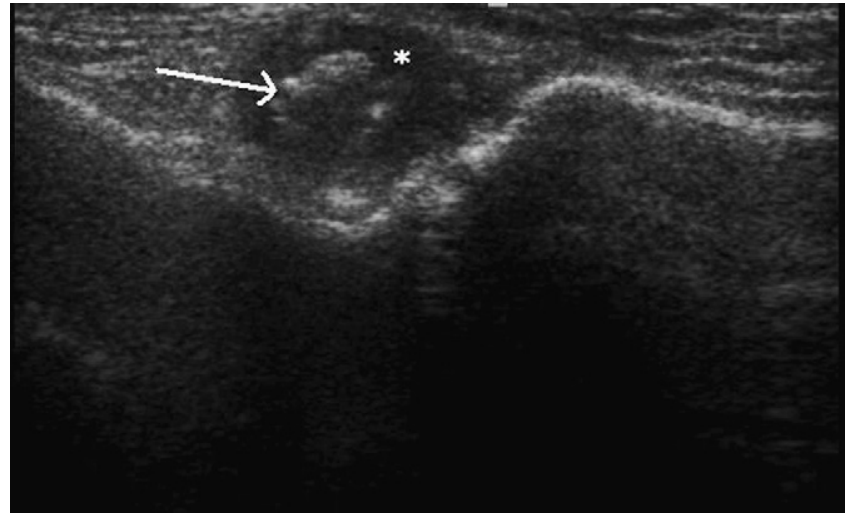

Fig. 25. Corticosteroid injection (methylprednisolone). Patient with a Psoriatic arthritis with thickened synovium $\left(^{*}\right)$. US shows an injection in the 2 nd metatarsophalangeal joint. Arrow indicates the air bubbling effect.

\subsection{Technical outcome}

The overall success rate was $100 \%(n=27)$. In all cases success was achieved with correct needle placement inside the joint. No complications occurred in the infusion or within follow-up period as vasovagal reactions or joint infection.

\subsection{Clinical outcome}

All patients $(n=27)$ improved their status solving swelling and tenderness, with improvement in VAS for pain. No short-term clinical adverse side effects were noted. No complications were recorded during the follow-up period of 6 months.

\section{Discussion/conclusion}

US-guidance is very reliable to afford a safety procedure always checking the injection, biopsy or aspiration. Guided-biopsy has high success rates obtaining several samples. Thus is also possible to safely use more powerful/long acting therapeutic drugs aggressive to extra-articular structures like Triamcinolone or Yttrium-90. In clinical terms, therapeutic procedures under US-guidance, are short-term useful and very safe options in persistent synovitis unresponsive to conventional therapy.

\section{Conflicts of interest/disclaimer}

The authors declare that the submitted article is not under consideration for publication elsewhere and that they have participated sufficiently in this study to take public responsibility for its content.

All the authors or their institutions have no conflicts of interest, financial or personal relationships that inappropriately influence their actions regarding this article.

\section{Acknowledgements}

We thank to Gracinda Costa, MD and Pedro Abreu, MD by their support in the Radiosynoviorthesis. We thank also to Lara Rodrigues, MD for the help in the provided illustrations.

\section{References}

[1] Jeyapalan K, Choudhary S. Ultrasound-guided injection of triamcinolone and bupivacaine in the management of De Quervain's disease. Skeletal Radiol 2009;38(November (11)):1099-103. 
[2] Jahangier ZN, Jacobs JW, van Isselt JW, et al. Persistent synovitis treated with radiation synovectomy using yttrium-90: a retrospective evaluation of 83 procedures for 45 patients. Br J Rheum 1997;36:861-9.

[3] Clunie G, Fischer M. EANM. EANM procedure guidelines for radiosynovectomy. Eur J Nucl Med Mol Imaging 2003;30(March (3)):BP12-6 [no abstract available].

[4] Román Ivorra J, Chismol Abad J, Morales Suárez Varela M, et al. Radioisotopic synoviorthesis in rheumatoid arthritis. A study of 108 cases. Rev Esp Med Nucl 2000;19:275-8.

[5] Kresnik E, Mikosch P, Gallowitsch HJ, et al. Clinical outcome of radiosynoviorthesis: a meta-analysis including 2190 treated joints. Nucl Med Commun 2002;23:683-8.

[6] Cruz-Esteban C, Wilke WS. Innovative treatment approaches for rheumatoid arthritis. Non-surgical synovectomy. Baillieres Clin Rheumatol 1995;9(November (4)):787-801.

[7] Sibbitt Jr WL, Peisajovich A, Michael AA, et al. Does sonographic needle guidance affect the clinical outcome of intraarticular injections? J Rheumatol 2009;36(September (9)):1892-902.
[8] Elentuck D, Palmer WE. Direct magnetic resonance arthrography. Eur Radiol 2004;14:1956-67.

[9] Valls R, Melloni P. Sonographic guidance of needle position for MR arthrography of the shoulder. Am J Roentgenol 1997;169:8457.

[10] Catalano OA, Manfredi R, Vanzulli A, et al. MR arthrography of the glenohumeral joint: modified posterior approach without imaging guidance. Radiology 2007;242:550-4.

[11] Lohman $M$, Vasenius $J$, Nieminen $O$. Ultrasound guidance for puncture and injection in the radiocarpal joint. Acta Radiol 2007;48:7447.

[12] Chung CB, Isaza IL, Angulo M, et al. MR arthrography of the knee: how, why, when. Radiol Clin North Am 2005;43:733-46. 